\title{
Monte Carlo Study of a Liquid-Liquid Phase Transition Using a Modified Gibbs Ensemble
}

\author{
D. A. Barlow \\ Alderman Barlow Labs, PO Box 1394 Trenton, Fla. 32693 USA \\ E-mail: doug.barlow@aldermanbarlow.com
}

\begin{abstract}
The carbon disulfide-methanol liquid-liquid critical point is studied using a Monte Carlo simulation of classical Stockmayer particles. A low energy configuration for the segregated two component system is determined using standard Monte Carlo methods then a modified Gibbs ensemble is employed to study the effect of transferring particles from one phase to another. Rather than use the model for the entropy of mixing in the Gibbs ensemble, which is of the regular solution type, a semi-quasi-chemical model is used which involves an interaction energy. We are able to simulate the mixing of the two components as the temperature approaches the critical temperature from below. Further, a method is given whereby the simulation results can be used to predict the critical temperature.
\end{abstract}

\section{Introduction}

A well known limitation in the simulation of systems of particles of finite size is the inability to reliably predict the occurrence of first and second order phase transitions; the so called finite size effect. Though there has been some success modeling phase transitions in Ising ferromagnets and the $q$-state Potts model [1], challenges remain in using computer models to predict phase transitions in bulk liquids and solids.

For bulk phase transitions in chemical systems an energetic description for the effect is given by the fact that at the transition temperature the chemical potentials for each of the two phases become equivalent. Given an accurate model for the chemical potential for each phase, the transition temperature can be predicted. However, an exact description of the chemical potential for many species, in various states, remains elusive. Recently, this approach was successfully used to predict the solid-solid structural phase transition temperatures within II-VI semiconductor alloys [2]. A very similar approach is taken here in the study of a liquid-liquid phase transition.

In certain binary liquid mixtures below a particular temperature, the critical temperature $T_{c}$, the two components are not miscible. Above the critical temperature the two phases readily dissolve into one another. A famous system of this sort is that of a mixture of the isotopes $\mathrm{He}^{3}$ and $\mathrm{He}^{4}[3]$. At about $0.8 \mathrm{~K}$ the two isotopes separate spontaneously into a nearly pure $\mathrm{He}^{3}$ phase and a $\mathrm{He}^{4}$ phase with a small amount of $\mathrm{He}^{3}$ dissolved in it. A well-known binary liquid system with a room temperature critical point is that of isobutyric acid and water [4]. In this work we have considered the $\mathrm{CS}_{2}$-methanol system. A liquid mixture of these two compounds has a convenient liquid-liquid critical point of $T_{c}=36^{\circ} \mathrm{C}$ at atmospheric pressure [5]. Below this temperature these liquids are separated into two distinct phases. Above $T_{c}$ the two compounds dissolve into one phase. At the critical temperature the phenomenon of critical opalescence 
occurs. That is, the normally clear liquid becomes opaque due to density fluctuations of sizes on the order of the wavelength of visible light. In this equilibrium simulation we make no attempt to reproduce these fluctuations but rather will be interested in temperatures where mixtures of the two components are favored over the situation of two distinct phases. Using a standard Monte Carlo method a segregated system of particles for this system is relaxed to an equilibrium energy near its estimated cohesive energy for a particular temperature. Then, using a modified version of the so called Gibbs ensemble $[6,7,8]$ the likelihood of the particles mixing is studied. This leads to a method for predicting the critical temperature of this system.

\section{Simulation Details}

The Monte Carlo scheme outlined by Mouritsen [9] is used here. A supercell of 256 particles is established with periodic boundary conditions. That is, ghost copies of the supercell surround the central cell on all sides. The initial configuration is $f c c$. The weight proportion used for the study of the $\mathrm{CS}_{2}$-methanol liquid-liquid critical effect is 20:80 methanol to $\mathrm{CS}_{2}$ as suggested by Gopal [5]. This proportion is approximately met by assigning half the particles to be methanol the other half $\mathrm{CS}_{2}$.

The classical pair potential used will be the one that is associated with the name of Stockmayer. Contained within this pair potential function is the repulsive term and the induced dipole attraction term of a Lennard-Jones potential plus an additional term for the interaction of static dipoles. In this representation the potential energy, $U$, between two particles is given by $[10]$

$$
U=4 \epsilon\left[\left(\frac{\sigma}{r}\right)^{12}-\left(\frac{\sigma}{r}\right)^{6}\right]-\frac{\mu_{1} \mu_{2}}{r^{3}} g\left(\theta_{1}, \theta_{2}, \phi_{2}-\phi_{1}\right) .
$$

Here $\sigma$ and $\epsilon$ are the standard Lennard-Jones parameters, $r$ is the inter-nuclear distance, $\mu_{i}$ the dipole moment of particle $i$ and $g$ is a function of the four angles $\theta_{1}, \theta_{2}, \phi_{2}$ and $\phi_{1}$ which describe the relative orientation of the dipoles for the two particles [11]. When using Eq. (1), with both particles not being the same species of molecule, the Lennard-Jones parameters are combined using the Lorentz-Berthelot mixing rules [12]. Parameters values used in the implementation of Eq. (1) are listed in Table 1.

Table 1. Parameter values used in the simulation. $\left(\mathrm{CO}_{2}\right.$ data used for $\left.\mathrm{CS}_{2}\right)$. $\dagger$ (Ref. [11] p. 1112).

\begin{tabular}{llll}
\hline & $\sigma(\AA)$ & $\epsilon(\mathrm{eV})$ & $\mu(\mathrm{Cm})$ \\
\hline $\mathrm{CS}_{2}$ & 3.794 Ref. [12] & 0.0194 Ref. [12] & - \\
methanol & $3.480 \dagger$ & $0.04372 \dagger$ & $5.671 \times 10^{-30}$ Ref. $[13]$ \\
\hline
\end{tabular}

Beginning with the supercell evenly divided between an upper methanol layer, and a lower $\mathrm{CS}_{2}$ layer, the system is moved to a low energy state via a Monte Carlo procedure. The initial lattice constant was chosen so that the system was initially at approximately $1.0 \mathrm{~kJ} / \mathrm{mol}$ above the cohesive energy at that temperature. Here we approximate the cohesive energy of the liquid as

$$
E_{c}=3 R\left(T_{m}-T\right)+\Delta H_{v a p},
$$

where $R$ is the gas constant and $T$ is absolute temperature. $T_{m}$ and $\Delta H_{v a p}$ are the melting temperature and the heat of vaporization for the more volatile species, in this case $\mathrm{CS}_{2}$, respectively. We considered temperatures from the critical point at $309 \mathrm{~K}$ on down to just above the methanol melting point i.e. $178 \mathrm{~K}$. Typically, within 7000 to 15000 Monte Carlo moves the system reaches the desired cohesive energy level. 
Next, the propensity for the particles to mix from one phase to the other is studied. This switching effect is examined using a modified form of the so called Gibbs ensemble. For a two component system the Gibbs ensemble is $[6,7]$ :

$$
W=\Delta E+k_{B} T \ln \frac{\left(V-V_{1}\right)\left(N_{1}+1\right)}{V\left(N-N_{1}\right)} .
$$

Here $k_{B}$ is Boltzmann's constant. In a two phase fluid $V_{1}$ is the volume of one phase, $V_{2}$ the other and $V=V_{1}+V_{2} . N_{1}$ is the number of component one particles, $N_{2}$ the number of the other and $N=N_{1}+N_{2} . \Delta E$ is the change in system energy resulting from the swapping of position for two particles of opposite type. For our case here Eq. (3) can be simplified since $V_{1} \simeq V_{2}, N_{1} \simeq N_{2}$ and $N_{1}>>1$. With these simplifications Eq. (3) reduces to

$$
W=\Delta E-k_{B} T \ln 2,
$$

From this point the switching moves are sampled according to the generalized Boltzmann factor $\exp \left[-W / k_{B} T\right]$.

Eq. (3) has the form of an isothermal free energy of mixing for a regular solution [14]. Here the second term on the right is then $T \Delta S_{\text {mix }}$ where $\Delta S_{\text {mix }}$ is an entropy of mixing. The regular solution model assumes complete randomness in mixing. That is, the entropy of mixing is that of an ideal mixture.

An entropy of mixing model that accounts for a temperature dependent preference of one type of bonding over the other is the so called quasi-chemical model (Ref. [14] pg. 38). In this scheme the geometric mean of the number of $\mathrm{AB}$ pairs in the mixture is weighted by $\exp \left[-w / k_{B} T\right]$ where $w$ is an interaction energy. With knowledge of $w$, the change in free energy upon mixing can be derived.

In this work we take a simplified approach in the spirit of the quasi-chemical model and let the entropy of mixing equal the regular solution entropy of mixing weighted by $\exp \left[-w / k_{B} T_{c}\right]$. So for a two component mixture of mole fraction $1 / 2$ this leads to

$$
\Delta S_{\text {mix }}=k_{B} \ln 2 \exp \left[-w / k_{B} T_{c}\right] .
$$

If $w>0$, the entropy of mixing will be less than that of the regular solution. At constant temperature, the Gibbs free energy of mixing $\Delta G_{m i x}$ is

$$
\Delta G_{m i x}=\Delta E-T \Delta S_{m i x} .
$$

If $\Delta G_{m i x}>0$ mixing is not favored, if $\Delta G_{m i x}<0$ mixing is spontaneous. Therefore at the critical temperature $T_{c}$ we have that $\Delta E-T_{c} \Delta S_{m i x}=0$. We can use this to relate $\Delta E$ to $w$. Solving for the entropy of mixing leads to

$$
\Delta S_{m i x}=\frac{\Delta E}{T_{c}} .
$$

Now this is equated with Eq. (5) and solving for $w$ yields

$$
w=-k_{B} T_{c} \ln \left(\frac{\Delta E}{k_{B} T_{c} \ln 2}\right) .
$$

Here we let $\Delta E$ be the change in system energy per particle resulting from the switching of one $\mathrm{A}$ and B pair each from its own phase in the still segregated simulation cell. From this, $w$ is computed. It is found for the binary system studied here that $w>0$.

A random switching move, from one phase to the other, almost always leads to $\Delta E>0$. The decision to accept the higher energy move is decided by comparing a random number between 0 
and 1 with $\exp \left[-w / k_{B} T\right]$. That is, the larger the magnitude of $w$ the less likely the possibility of the higher energy move being accepted.

Attempts at switching particles are made according to the prescription discussed above. Once a switch is accepted this event is tabulated and the system is returned to its original low energy state. Once a pair is successfully switched it is taken from the set of future possible switches. For purposes of visualization no more than 64 successful switches are allowed at the highest temperature considered. This is typically accomplished with approximately 525 switching attempts. Then, for all lower temperatures considered, the maximum number of switching attempts is set at 525 as well. For purposes of visualization, the location of accepted switched pairs is saved for each temperature considered. Then, selected configurations showing switched pairs are depicted for four temperatures between the melting temperature of methanol and the critical temperature for the $\mathrm{CS}_{2}$-methanol system.

\section{Results}

The simulation was carried out for eight temperatures between $178 \mathrm{~K}$ and $309 \mathrm{~K}$. At each temperature the simulation was repeated ten times. The values for $w$ and $\Delta E$ were found to be independent of temperature with mean values of $0.061 \mathrm{eV}$ and $0.00187 \mathrm{eV}$ respectively. In Figures 1 through 4 are depicted the supercell after the MC relaxation and switching for four representative temperatures for the liquid-liquid system leading up to the critical temperature at $309 \mathrm{~K}$. In Figure 5 the average number of accepted switching moves vs. temperature, for the eight temperatures from $178 \mathrm{~K}$ to $309 \mathrm{~K}$, are plotted.

In Figure 6 a sketch is given of $\Delta G_{m i x}$ vs. $T$ as given by Eqs. (5) and (6) using the values for $w$ and $\Delta E$ mentioned above. The free energy of mixing is positive for low temperatures, goes to zero at the critical temperature then to negative for higher temperatures. One can see how a system say oil and water, would have a positive free energy of mixing over all relevant temperatures and thus never mix while a system like water and methanol would have a negative free energy of mixing over all relevant temperatures and thus never be observed to separate into two distinct phases.

Here the known critical temperature was used in the determination of the interaction energy $w$. However, given a model for the interaction energy, Eq. (8) could then be used to directly compute the critical temperature. Guggenheim suggests that $2 w / z$ should equal the energy to take one $\mathrm{A}$ dimer and one $\mathrm{B}$ dimer and create two $\mathrm{AB}$ molecules, where $z$ is number of nearest neighbors (Ref. [14] p. 38). We can write this as

$$
\frac{2 w}{z}=2 E_{A B}-E_{A A}-E_{B B} .
$$

Using data from Table 1 along with our Stockmayer potential given by Eq. (1) we can estimate the energies in Eq. (9) and determine a value for $w$. The binding energy is computed for the three cases: AB, AA and BB. The dipoles for two methane molecules are paired so as to give the lowest dipole energy configuration and likewise all internuclear distances are set to give the lowest Lennard-Jones energy. Computing and substituting these values into Eq. (9) yields: $2 w / z=0.032556 \mathrm{eV}$. Assuming that $z=4$ we find that $w=0.065112 \mathrm{eV}$. Using this value for $w$, along with $\Delta E$ from above in Eq. (8), leads to $T_{c} \simeq 323 \mathrm{~K}$. This similarity with the known value for $T_{c}$ is significant and further investigation is warranted. 


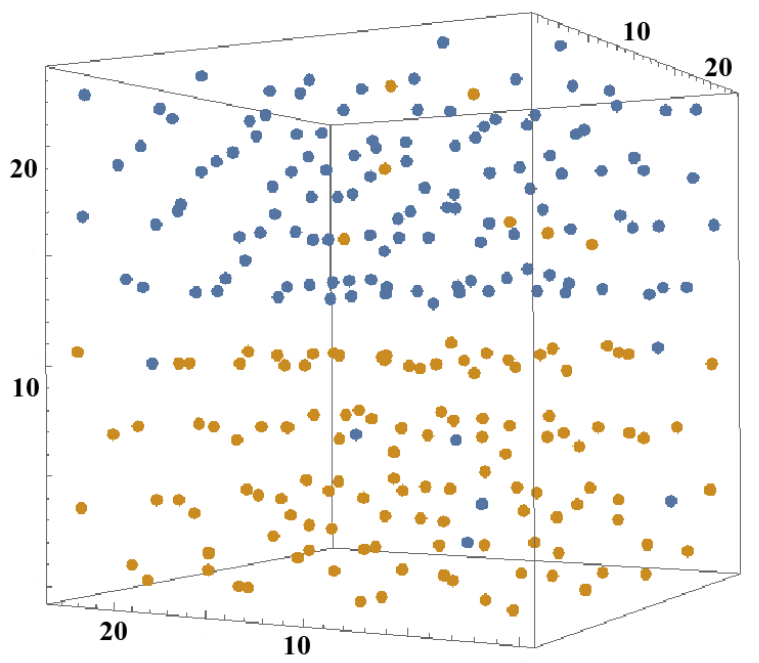

Figure 1. Supercell of 256 molecules in a simulated liquid mixture after $\mathrm{MC}$ relaxation and particle switching at $178 \mathrm{~K}$. Amber dots represent $\mathrm{CS}_{2}$ while blue ones denote methanol. Distances are in angstroms.

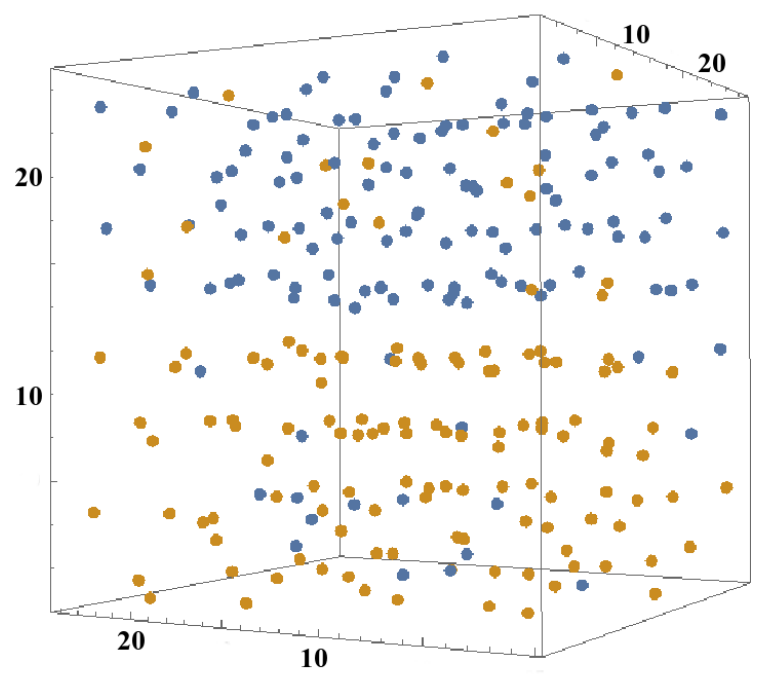

Figure 2. Supercell of 256 molecules in a simulated liquid mixture after $\mathrm{MC}$ relaxation and particle switching at $210 \mathrm{~K}$.

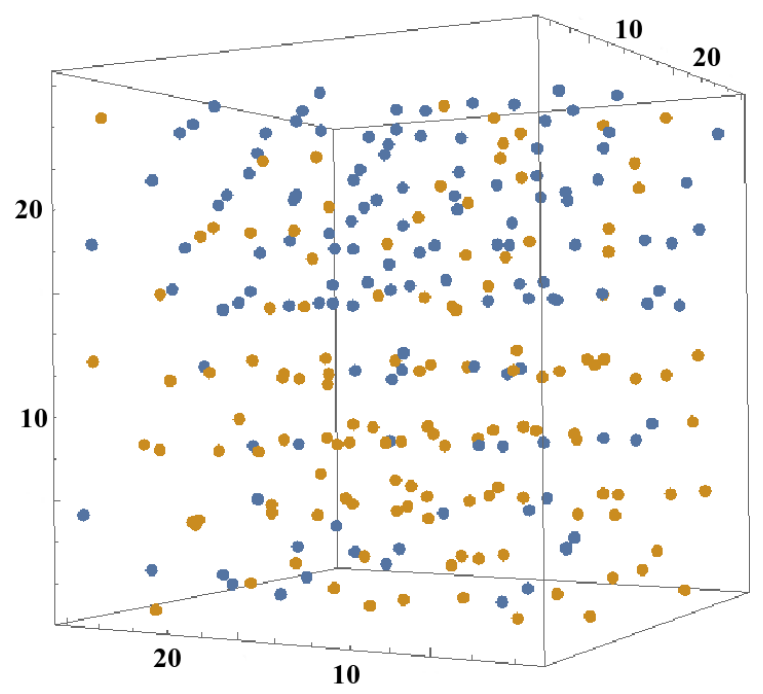

Figure 3. Supercell of 256 molecules in a simulated liquid mixture after MC relaxation and particle switching at $270 \mathrm{~K}$.

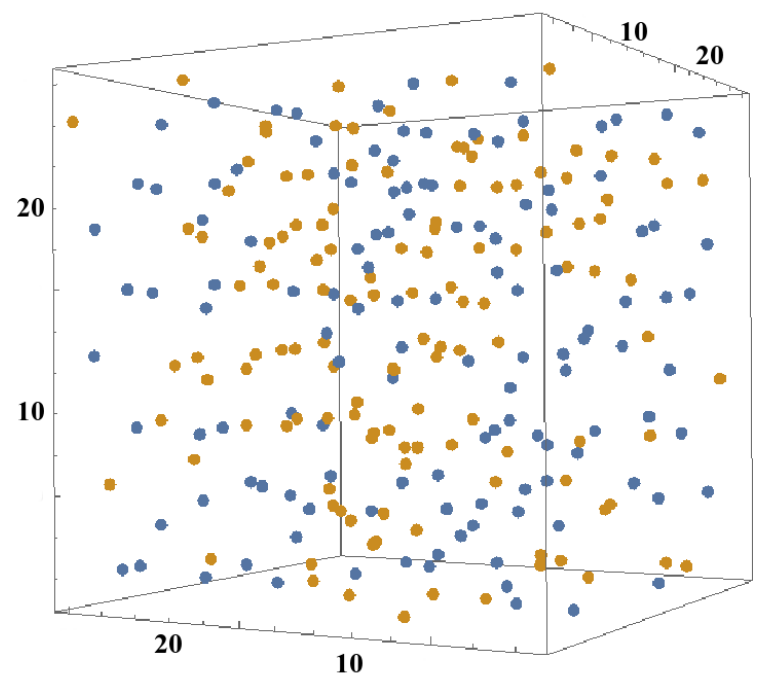

Figure 4. Supercell of 256 molecules in a simulated liquid mixture after $\mathrm{MC}$ relaxation and particle switching at the critical temperature of $309 \mathrm{~K}$. 


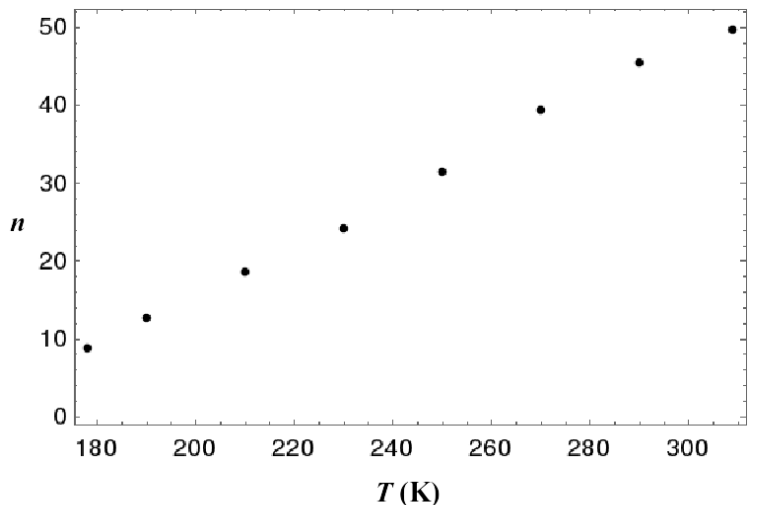

Figure 5. Number of accepted pair switches $n$ vs. temperature.

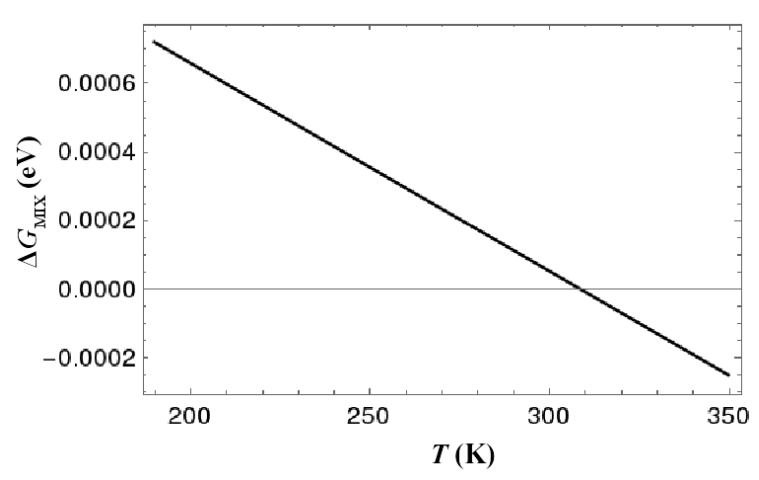

Figure 6. Estimated free energy of mixing per particle for the $\mathrm{CS}_{2}$-methanol system.

This study points to several items for future work. Repeating these simulations using threebody potentials $[15,16]$ for $\mathrm{CS}_{2}$ and methanol should be considered. Other important binary liquids that display this type of transition could be analyzed as in this report. Such systems include ${ }^{3} \mathrm{He} /{ }^{4} \mathrm{He}$, isobutyric acid-water and methanol-cyclohexane. Additionally, the full quasichemical treatment, where the entropy of mixing is a function of temperature and an interaction energy, should be considered.

\section{Acknowledgement}

The author wishes to thank Prof. Jim Baird for helpful discussions.

\section{References}

[1] Binder K 1992 The Monte Carlo Method in Condensed Matter Physics ed K Binder (Berlin: Springer-Verlag) chapter 1 p. 4

[2] Barlow D A 2013 J. Phys. Chem. Solids 74 (3) 406

[3] Gasser R P H, Richards W G 1974 Entropy and Energy Levels (Oxford: Clarendon Press) pp. 83-84

[4] Geisy T J, Chou A S, McFeeters R L, Baird J K, Barlow D A 2011 Phys. Rev. E 83061201

[5] Gopal E S R 2000 Resonance 5 (4) 37

[6] Panagiotopoulos A Z 1987 Mol. Phys. 61813

[7] Levesque D, Weiss J J 1992 The Monte Carlo Method in Condensed Matter Physics ed K Binder (Berlin: Springer-Verlag) chapter 6 p. 125

[8] Bourov G K, Bhattacharya A 2007 J. Chem. Phys. 127244905

[9] Mouritsen O G 1984 Computer Studies of Phase Transitions and Critical Phenomena (Berlin: Springer-Verlag) chapter 2 p. 14

[10] Watts R O, McGee I J 1976 Liquid State Chemical Physics (New York: John Wiley \& Sons) p. 220

[11] Hirschfelder J O, Curtiss C F, Bird R B 1967 Molecular Theory of Gases and Liquids (New York: John Wiley \& Sons) p. 27

[12] Naserifar S, Tsotsis T T, Goddard III W A, Sahimi M 2015 J. Membr. Sci. 47385

[13] Lide D R 2008 Handbook of Chemistry and Physics $88^{\text {th }}$ Ed. (Boca Raton: CRC Press)

[14] Guggenheim E A 1952 Mixtures (Oxford: Clarendon Press) p. 26

[15] Koslowski T 1999 Zeit. Phys. Chem. Bd. 210 S 45

[16] Pálinkás G, Hawlicka E, Heinzinger K 1987 J. Phys. Chem. 914334 